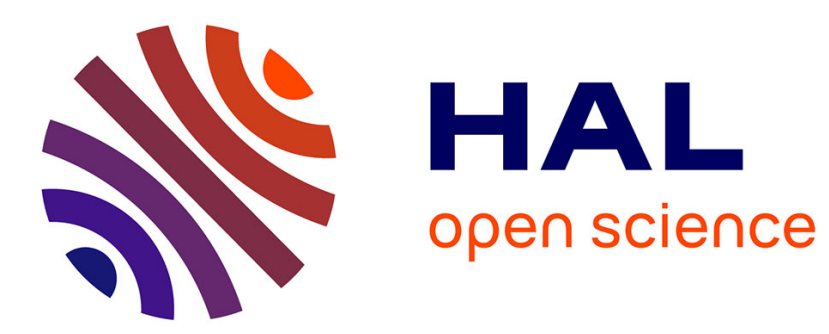

\title{
Waste management and household effort: toward an enhanced ADF policy
}

\author{
Stéphanie Lemaitre, Hubert Stahn
}

\section{To cite this version:}

Stéphanie Lemaitre, Hubert Stahn. Waste management and household effort: toward an enhanced ADF policy. 2009. halshs-00449537

\section{HAL Id: halshs-00449537 https://shs.hal.science/halshs-00449537}

Preprint submitted on 22 Jan 2010

HAL is a multi-disciplinary open access archive for the deposit and dissemination of scientific research documents, whether they are published or not. The documents may come from teaching and research institutions in France or abroad, or from public or private research centers.
L'archive ouverte pluridisciplinaire HAL, est destinée au dépôt et à la diffusion de documents scientifiques de niveau recherche, publiés ou non, émanant des établissements d'enseignement et de recherche français ou étrangers, des laboratoires publics ou privés. 


\section{GREQAM}

Document de Travail

Groupement de Recherche en Economie Quantitative d'Aix-Marseille - UMR-CNRS 6579 Ecole des Hautes Etudes en Sciences Sociales Universités d'Aix-Marseille II et III

\section{WASTE MANAGEMENT} AND

\section{HOUSEHOLD EFFORT:}

Toward an enhanced ADF policy

\section{Stéphanie LE MAITRE} Hubert STAHN

November 2009 


\title{
Waste Management and Household Effort : Toward an enhanced ADF policy
}

\author{
Stéphanie LE MAITRE and Hubert STAHN ${ }^{1}$ \\ GREQAM $^{2}$, University of the Mediterranean (Aix-Marseille II)
}

\footnotetext{
${ }^{1}$ We want to thank the participants of the "Journées Louis-André Gérard-Varet 08" and of the "Public Goods, Public Projects, Externalities (PGPPE)" workshop 08 on Environmental Economics for helpfull comments. We also acknowledge the financial support of the ANR PRECODD project CleanWasT.

${ }^{2}$ Groupement de Recherche en Economie Quantitative d'Aix-Marseille, UMR 6579 of the CNRS
} 


\begin{abstract}
Advanced Disposal Fees (ADF) are rather popular in waste management policies, but they cease any waste reduction effort at the household level. We propose a waved ADF policy which enhances this system by giving to the households the possibility to sign a waste reduction contract in counterpart of a lower fee. These contracts satisfy an incentive, a budget balancing and a participation constraints. For these feasible contracts, we show that this enriched ADF policy is welfare improving and always induces a waste reduction effort.
\end{abstract}

Keywords: Waste Management, Disposal Fee Policy, Household Effort, Contracts J.E.L. classification numbers: Q28, H21

Address:

LE MAITRE Stéphanie G.R.E.Q.A.M.

Université de la Méditerranée

Château Lafarge

Route des Milles

13290 Les MILLES

FRANCE

tel +33442935980

fax +33442930968

E-mail: stephanie.le-maitre@etumel.univmed.fr
STAHN Hubert (*)

G.R.E.Q.A.M

Université de la Méditerranée

Château Lafarge

Route des Milles

13290 Les MILLES

FRANCE

tel +33442935981

fax +33442930968

E-mail:stahn@univmed.fr

(*) Corresponding author 


\section{Introduction}

For the last few years, environmentalists and policy makers have focused on increasing attention to the question of waste management (see for instance Jenkins [9], Dinan [5] or Fullerton and Kinnanan [6]) and it is now largely recognized that consumption generates an increasing amount of garbage, the handling of which induces a growing social cost. From that point of view, it becomes obvious that the households, acting as Citizens, should participate in the waste management programs or at least have enough incentives to do so. In fact, even if this activity is more or less efficient according to the commodity under consideration (see Palmer and al [12] or Jenkins and al [10]), it is always cost reducing especially if the agents are heterogeneous with respect to the evaluation of the cost of their effort. This conviction largely motivated the per bag pricing (see Fullerton and Kinnanan [7]) and is essential for policies which one bets on residential curbside recycling programs or promote a policy for green design (see for instance Fullerton and $\mathrm{Wu}$ [8], Walls and Palmer [15], Calcott and Walls [1], [2]).

Yet, the behavior of the households is not often directly observable. This frequently induces inefficiency and leads to second best policies. To be more precise, if we refer for instance to the work of Choe and Fraser[3], [4] or Shinkuma[13], it is clear that a Disposal Fee (DF for short) policy, i.e. a charge based on the amount of waste, increases the risk of illegal dumping or at least requires the introduction of an incentive scheme which prevents this unpleasant event. In France, this type of financing for waste management which consists in making pay the user according to the quantity of waste that he throws, starts to develop: about twenty of localities (according to ADEME) adopted this kind of incentive fee. The effects, in these localities, are an increase in the efforts of sorting as well as an increase in the quantities of waste that can be recycled at the expense of residual waste. Moreover, uncivil behaviours do not seem significant and are obviously limited in time but not ineluctable.

However, in order to avoid the perverse effects associated with this type of "pay as you throw" financing, the policy makers can decide to directly include the waste management cost in the price of the commodity by implementing an Advanced Disposal Fee (ADF for short) policy. For instance the European Directive (2002/96/CE) institutes the ADF system on electrical and electronic equipment and prompts all European countries to transpose it in her national law systems like in France by the decree $n^{\circ} 2005-829$ of July 20th, 2005. Indeed, in France, since November 15, 2006, 4 eco-organizations deal with removal and treatment of electric and electronic equipments collected selectively, for producers who adhered to them. Consumers can bring back to the distributors used equipment when they purchase a new one. However, for the purchase of new equipment (refrigerators, computers, mobile phone...), the consumers must pay an "eco-contribution" intended to cover the costs of the new operations of collection and 
recycling of this waste. This amount can vary, for example, from one-cent euro for a mobile phone to several euros for a television or a computer. A label, fastened on each new material, indicates the cost of its recycling.

This suspicion which motivates the ADF system however definitely annihilates any incentives, at a household level, to participate to a waste management program. This is for instance not the case with a DF policy associated, when available, to a deposit-refund system. It even prevents illegal disposal (see Palmer and Walls [11]). For the more general class of durable goods, Shinkuma [14] still argues that an ADF policy is less efficient than a DF one. In this case, an ADF policy reduces the incentives to repair the commodity and depresses the second hand market. It follows an excessive consumption and a larger disposal cost in respect to the social optimum.

Our argument against a crude ADF policy is however slightly different. We simply say that if some households are willing to participate in a waste management program and perhaps obtain some financial compensation, we should not destroy these incentives by implementing a crude ADF system. On the contrary, we should implement a system which enriches this one by waving the disposal fee in counterpart of the waste reduction effort. We call this a waved ADF system (wADF) and we argue that such a wADF contract induces a double social gain especially when the agents are heterogeneous with respect to their desutility of their effort and their willingness to pay.

In fact, it is immediate that the buyers for which the desutility induced by the waste reduction effort is compensated by lower acquisition cost of the commodity surely participate in the waste reduction program and even improve her situation. But we can still go a step further by observing that any price cut in counterpart of a waste management effort gives the opportunity to new consumers to enter the market. Loosely speaking, "poorer and environment friendly" consumers, i.e. characterized by a lower willingness to pay and a small desutility of waste reduction effort, are, under a wADF system, able to have access to the good under consideration. This again increases the welfare.

The reader surely objects that everybody would have an incentive to accept this waste reduction contract, to take the money and to dump her waste illegally. This is why we also introduce a monitoring policy which checks the execution of the terms of the contract and we show that even under this incentive constraint our wADF policy is more efficient than a standard ADF system.

To be more precise, we take a standard ADF system and we give to the consumers the opportunity to sign a waste management contract. Consumers who accept the contract will 
be equipped with dustbins of different colours to sort their waste. We could also imagine, as it is the case in some French cities like those of the "Communauté de communes de la Porte d'Alsace", than these bins are provided with a electronic chip which allow to weigh the amount of the waste contained in these bins. Thus, this one prescribes on the one hand a decrease in the amount of waste in counterpart of price cut on their consumption but, on the other hand, relies on a monitoring policy characterized by a probability of control and a fine which is equal to the social cost of illegal dumping. The main purpose of this paper is to design this contract in a way to maximize the welfare and to show that this wADF system strictly dominates a crude $\mathrm{ADF}$ one.

But in our second best world, all contracts of that type cannot be implemented. That is why we restrict our attention to a subset of feasible contracts which are (i) budget balancing in the sense that the global waste management costs are covered, (ii) satisfy a participation constraint, i.e. at least one agent is willing to adopt the contract and (iii) incentive compatible, i.e. induces no illegal dumping. After having identified these feasible contracts, we study their properties in term of welfare.

We essentially show that a social planner always has an incentive to set the required waste treatment rate as high as possible and to keep the probability of control as small as possible in order to limit the monitoring cost. The first result is essentially linked to the entrance of "poorer and environment friendly" consumers on the market when a wADF is implemented while the second is induced by the budget constraint, i.e. a raise in the probability of control increases the monitoring cost, hence decreases the price cut. These results are obtained with only few restrictions on the distribution of both the willingness to pay and the desutility of the effort through agents.

These preliminary results allow us in a second step to characterize an optimal wADF policy. We show that this system always induces a reduction of the waste management cost independently from the monitoring cost and that the optimal policy follows from an arbitrage between the welfare gain relative to a rise in the required waste treatment rate and the increasing monitoring costs. The optimal policy is also related to the average cost of the effort in the population.

The paper proceeds as follows : the next section depicts our basic assumptions and describes the wADF system. In section 3, we present the restriction on the set of contracts imposed by the incentive, budget balancing and participation constraints. In section 4, we associate to each feasible contract its level of welfare and give some basic properties of this function. Section 5 is devoted to construction of the optimal contract. Finally, the last section contains some concluding remarks. The proofs of the different results are relegated to an appendix. 


\section{The basic assumptions and the wADF system}

In order to illustrate this question, we consider a commodity produced by a representative firm and sold to a continuum of consumers. This consumption produces a certain amount of waste. This end-of-pipe pollution can be, as in Choe and Fraser ([3]), considered as unit cost $c$ while the free individual disposal cost is of $c_{d}$ but it induces a social cost of $c_{s}$. Of course we have $0<c_{d}<c<c_{s}$.

Since we mainly focus on the consumer behavior, we assume that (i) there is no way to reduce the intrinsic waste content at the production level, (ii) waste can be recycled even partially and (iii), one unit of good produces, to simplify, one unit of waste. We also largely simplify the behavior of the representative firm. This one produces the commodity at a zero constant unit. This means in other words that the competitive price $p$ is under a standard ADF system equal to the waste treatment cost $c$.

\subsection{The demand side}

The demand side is more detailed. We consider a continuum of heterogeneous consumers who decide to buy or not the good, i.e. $x \in\{0,1\}$ and choose their intensity of effort $e \in[0,1]$ in waste downsizing. They share the same utility function $u(x, e, m)=\alpha x-\theta e-m$ where $m$ denotes the monetary spending. Nevertheless, they are heterogeneous in respect to their willingness to pay $\alpha \in[0, A]$ and their marginal cost $\theta \in[0, \Theta]$ of the waste reduction effort. Moreover, in order to make sure that at least one agent is able to consume when the waste management cost is pre-paid, we assume that $A>c$. In the same vein, we say that $\Theta>c$ otherwise all consumers are willing to provide an effort.

The distribution of these two characteristics across the population is summarized by a probability distribution over $[0, A] \times[0, \Theta]$ whose cumulated distribution function is denoted $F(\alpha, \theta)$. This c.d.f. is assumed to be absolutely continuous with a strictly positive density $f(\alpha, \theta):=\partial_{\alpha, \theta}^{2} F(\alpha, \theta)>0$. Moreover we denote by $f(\alpha, \cdot):=\int_{0}^{\Theta} f(\alpha, \theta) d \theta$ the marginal density of $\alpha$ and by $f(a / \theta):=\frac{f(\alpha, \theta)}{f(\cdot, \theta)}$ the conditional density of $\alpha$ given $\theta$. A symmetric interpretation holds for $f(\cdot, \theta)$ and $f(\theta / \alpha)$.

Now remember that effort reduces waste. We measure the outcome of this activity by the proportion $r(e)$ by which the unit waste treatment cost is reduced ${ }^{1}$. We however assume that

\footnotetext{
${ }^{1}$ This assumption particulary fits well in our discrete choice model since a consumer buys at most one unit and therefore induces a waste treatment cost of at least $c$. Otherwise one should take into account the amount of good consumed.
} 
there is an upper bound $\bar{r} \leq 1$ to this proportion and that this relation is linear, i.e. $r(e)=\bar{r} \cdot e$ for $e \in[0,1]$. The largest waste management cost reduction is obtained when the intensity of the effort is maximal. From that point of view we can say that $e(r)=\frac{r}{\bar{r}}$ denotes the level of effort required to reduce the waste management cost in a proportion of $r \in[0, \bar{r}]$. Moreover we assume for technical reason that $\frac{c_{d}}{c}<\bar{r}$ otherwise the problem becomes trivial since, as we will see it later, the optimal policy sets $r=\bar{r}$.

\subsection{The wADF system}

Within this setting a policy maker has basically two options. She can implement a crude ADF policy by charging in advance a fee of $c$ per unit of commodity sold by the firm. In this case, she covers her waste treatment cost and prevents illegal dumping whose social cost $c_{s}$ is higher than $c$. However she also dissuades all waste reduction effort within the set of households which are willing to buy the good at price $c$ and even excludes from consuming a subset of agents which are ready to make a waste reduction effort in compensation of a price cut.

That is why we propose, in this paper, a waved Advance Disposal Fee (wADF) system. In fact we give the policy maker the possibility to associate to the purchase of the good a contract which specifies, when it is accepted, a target $r$ of waste management cost reduction associated to a price discount $d$ for the buyer. This however opens the door to illegal dumping, thus we couple this contract with a monitoring strategy. We denote by $\pi$ the probability that a consumer who accepts the contract is controlled and by $c(\pi)$ the unit cost of a control. The fine is set to the social cost $c_{s}$ of illegal deposit. Moreover we assume that this control cost is increasing and convex (i.e. $c^{\prime}(\pi)>0$ and $c^{\prime \prime}(\pi)<0$ ) and that the absence of monitoring is costless (i.e. $c(0)=0$ ) while perfect motoring is very expensive (i.e. $c(1)>c$ ).

The reader surely observed that a contract characterized by $(r, d, \pi)=(0,0,0)$ coincides to a standard ADF contract. The question in this second best world therefore becomes : is there another contract which is better (i.e. which maximizes the surplus) and which (i) balances the budget of the policy maker, (ii) will be accepted by at least some agents, (iii) provides enough incentives to prevent illegal dumping?

\subsection{The choice of a consumer}

These restrictions on the set of available contracts are studied in the next section; but in order to fix more notations, let us quickly come back to the set of alternatives which are open to the households when a wADF system is implemented. 
In our discrete choice model, either a consumer of type $(\alpha, \theta)$ buys nothing and her utility is nought. If she purchases the good, she can refuse $(\bar{w})$ the waste reduction contract and stay in a standard ADF. In this case, she pre-pays the cost $c$ of waste disposal and has no incentive to make an effort. Under our zero marginal production cost assumption, she pays $p=c$ for the good and her utility is given by :

$$
u_{\bar{w}}^{(\alpha, \theta)}=\alpha-c
$$

If she accepts the contract, she obtains a discount of $d$, but she always has the opportunity either to execute $(e)$ or not $(\bar{e})$ the terms of the contract. In the first case she makes the required effort and delivers the transformed waste. Her utility is therefore given by :

$$
u_{w e}^{(\alpha, \theta)}(r, d)=\alpha-(c-d)-\theta e(r)
$$

Otherwise, she makes no effort and in order to mask her infringement she dumps her waste illegally at private cost $c_{d}$. She nevertheless takes the risk of being caught with probability $\pi$ and of being charged of a fine that we have set at the social cost $c_{s}$ of illegal dumping. She therefore obtains :

$$
u_{w \bar{e}}^{(\alpha, \theta)}(d, \pi)=\alpha-(c-d)-c_{d}-\pi c_{s}
$$

From that point of view, the best strategy of a consumer of type $(\alpha, \theta)$ is the one which gives her the highest payoff, i.e. which satisfies

$$
\max \left\{0, u_{\bar{w}}^{(\alpha, \theta)}, u_{w e}^{(\alpha, \theta)}(r, d), u_{w \bar{e}}^{(\alpha, \theta)}(d, \pi)\right\}
$$

\section{The set of feasible contracts}

The main purpose of this section is to construct the contracts which can be proposed by the policy maker and which have the property that (i) she covers both the waste management and the monitoring costs, (ii) at least one agent is willing to participate in the waste reduction program, and (iii) all agents who accept the contract have enough incentives to implement it, i.e. do not use an illegal dumping strategy.

The first constraint, as we will see it soon, dictates the price cut $d$ conceded to the consumers. That is why we denote by $\mathcal{C}=\{(r, \pi) \in[0, \bar{r}] \times[0,1]\}$ the set of available contracts.

\subsection{The price cut and the Budget Balancing constraint}

If the requirements (ii) and (iii) are both satisfied, it becomes very easy to compute the price cut $d$ induced by a budget balancing condition. To perform this computation, let us denote by $P_{w e}$ 
the proportion of consumers who accept and execute the contract and by $P_{\bar{w}}$ the proportion of households who keep a standard ADF system. With this convention, and since we have assumed that there is no illegal disposal (i.e. (ii) holds), the policy maker collects the following per capita amount of money:

$$
t=P_{\bar{w}} \cdot c+P_{w e} \cdot(c-d)
$$

This tax covers the waste management and the monitoring costs. If there is no illegal disposal, the per capita waste management costs are respectively of $P_{w e} \cdot(1-r) \cdot c$ and of $P_{\bar{w}} \cdot c$ for the households who accept or not the waste reduction contract. Under this same assumption, the monitoring costs are pure sunk cost since no fine is collected. If we have in mind that a control is charged at $c(\pi)$ and that a proportion $\pi$ of the households accepting the contract is monitored, the per capita spending is given by :

$$
s=P_{\bar{w}} \cdot c+P_{w e} \cdot(1-r) \cdot c+\left(\pi \cdot P_{w e}\right) \cdot c(\pi)
$$

A simple budget balancing constraint therefore shows that :

Lemma 1 If there is no illegal dumping the budget balancing price cut is given by $d=r \cdot c-\pi \cdot c(\pi)$

So, let us now rewrite $u_{w e}^{(\alpha, \theta)}(r, d)$ and $u_{w \bar{e}}^{(\alpha, \theta)}(d, \pi)$ as respectively :

$$
\left\{\begin{array}{l}
u_{w e}^{(\alpha, \theta)}(r, \pi)=\alpha-(1-r) \cdot c-\pi \cdot c(\pi)-\theta e(r) \\
u_{w \bar{e}}^{(\alpha, \theta)}(r, \pi)=\alpha-(1-r) \cdot c-\pi \cdot c(\pi)-c_{d}-\pi c_{s}
\end{array}\right.
$$

\subsection{The Participation Constraint}

By this constraint, we mean that the contract is built in a way which ensures that at least one buyer is willing do adopt and execute the contract. This means more formally that :

$$
\exists(\alpha, \theta) \in[0, A] \times[0, \Theta], \quad u_{w e}^{(\alpha, \theta)}(r, \pi)=\max \left\{0, u_{\bar{w}}^{(\alpha, \theta)}, u_{w e}^{(\alpha, \theta)}(r, \pi), u_{w \bar{e}}^{(\alpha, \theta)}(r, \pi)\right\}
$$

Moreover if the incentive constraint is satisfied, i.e. the strategy $w \bar{e}$ is never chosen, this condition reduces to :

$$
\exists(\alpha, \theta) \in[0, A] \times[0, \Theta], \quad u_{w e}^{(\alpha, \theta)}(r, \pi) \geq \max \left\{0, u_{\bar{w}}^{(\alpha, \theta)}\right\}
$$

Since $A>c$, we know that the quantity $u_{\bar{w}}^{(\alpha, \theta)}$ is positive for a set of agents which is dependent on $\theta$. This simply follows from the fact that the market is always active even in a standard ADF system. So if the price cut $d=r \cdot c-\pi \cdot c(\pi)$ is positive, we can identify in this previous set at least one agent with a sufficiently small $\theta$ who is willing to participate in the waste 
reduction program. The positivity of the price cut is therefore a sufficient condition to meet the participation constraint but, more interestingly, it is also a necessary one. In fact we prove that:

Lemma 2 If there is no illegal dumping, the condition $(P C)$ is equivalent to $d=r \cdot c-\pi \cdot c(\pi) \geq 0$, a non negative budget balancing price cut.

\subsection{The Incentive Constraint}

In this case, we have to make sure that the parameters which characterize the contract preclude illegal waste dumping. In other words, we want that each household always prefers either to execute the contract, to pre-pay the waste management or even not to consume. This means more formally that:

$$
\forall(\alpha, \theta) \in[0, A] \times[0, \Theta], \quad \max \left\{u_{w e}^{(\alpha, \theta)}(r, \pi), u_{\bar{w}}^{(\alpha, \theta)}, 0\right\} \geq u_{w \bar{e}}^{(\alpha, \theta)}(r, \pi)
$$

This constraint is, of course, satisfied when the price cut $d=r \cdot c-\pi \cdot c(\pi)$ associated to the waste management contract is lower than the total cost induced by illegal dumping, $t c=c_{d}+\pi \cdot c_{s}$ including both the individual disposal cost and the average fine. In fact, if this condition is true, a pre-payment system is always preferable to illegal dumping, i.e. $u_{\bar{w}}^{(\alpha, \theta)} \geq u_{w \bar{e}}^{(\alpha, \theta)}(r, \pi)$. It follows by the definition of a maximum that (IC) is satisfied. Yet, we can again go a step further by showing that this condition is not only sufficient but also necessary.

Lemma 3 The (IC) condition is equivalent to $r \cdot c-\pi \cdot c(\pi) \leq c_{d}+\pi \cdot c_{s}$, an upper bound on the budget balancing price cut.

At that point, we are now able to precisely characterize the subset $\mathcal{F} \subset \mathcal{C}$ of feasible contracts satisfying simultaneously the budget balancing, the participation and the incentive constraints.

Proposition 1 The set $\mathcal{F} \subset \mathcal{C}$ of feasible contracts is non empty and is given by :

$$
\mathcal{F}=\left\{(r, \pi) \in[0, \bar{r}] \times[0,1]: \frac{\pi \cdot c(\pi)}{c} \leq r \leq \frac{c_{d}}{c}+\frac{\pi \cdot\left(c(\pi)+c_{S}\right)}{c}\right\}
$$

It remains now to select in this set of feasible contracts the one which maximizes the average surplus, but in order to address this question let us first compute this quantity for all feasible contracts and let us identify their properties. 


\section{The surplus and its basic properties}

In order to underline the double gain induced by a wADF system, we conduct this computation in two steps. We first consider the households who buy the good in both an ADF or a wADF system and show that these agents do not get worse after the introduction of a wADF. Then, we move to the consumers who really gain from a wADF, that is the "poorer and environment friendly" agents who gain access to the good by obtaining a price cut in compensation of their effort. The last step of this section is devoted to the properties of the total surplus.

The first population is quite easy to identify since there willingness $\alpha$ to pay must be greater than $c$, the competitive price charged in an ADF system. If an incentive compatible contract is available, the subset of agents who accepts is typically given by :

$$
C(r, \pi)=\left\{(\alpha, \theta) \in[0, A] \times[0, \Theta]: \alpha \geq c \text { and } u_{w e}^{(\alpha, \theta)}(r, \pi) \geq u_{\bar{w}}^{(\alpha, \theta)}\right\}
$$

A simple computation shows that the second condition is equivalent to $\theta e(r) \leq c \cdot r-\pi \cdot c(\pi)$ with $e(r)=\frac{r}{\bar{r}}$. This implies that $\theta \leq \theta(r, \pi):=\bar{r}\left(c-\frac{\pi \cdot c(\pi)}{r}\right)$. We can therefore say that ${ }^{2}$ :

$$
C(r, \pi)=\{(\alpha, \theta) \in[0, A] \times[0, \Theta]: c \leq \alpha \leq A \text { and } 0 \leq \theta \leq \theta(r, \pi)\}
$$

By complementation, we also observe that the households who buy the good in an ADF system but reject the contract are given $b^{3}{ }^{2}$

$$
\bar{C}(r, \pi)=\{(\alpha, \theta) \in[0, A] \times[0, \Theta]: c \leq \alpha \leq A \text { and } \theta(r, \pi) \leq \theta \leq \Theta\}
$$

It follows that the surplus of these two categories of, say Initial households, is given by :

$$
\begin{aligned}
S_{I}(r, \pi) & =S_{C}(r, \pi)+S_{\bar{C}}(r, \pi)=\int_{C(r, \pi)} u_{w e}^{(\alpha, \theta)}(r, \pi) d F+\int_{\bar{C}(r, \pi)} u_{\bar{w}}^{(\alpha, \theta)} d F \\
& =\int_{c}^{A}\left(\int_{0}^{\theta(r, \pi)} u_{w e}^{(\alpha, \theta)}(r, \pi) f(\theta / \alpha) d \theta+\int_{\theta(r, \pi)}^{\Theta} u_{\bar{w}}^{(\alpha, \theta)} f(\theta / \alpha) d \theta\right) f(\alpha, \cdot) d a
\end{aligned}
$$

and we can even observe that :

\footnotetext{
${ }^{2}$ The reader surely observed that (i) since $c<\Theta$ and $\bar{r} \in[0,1]$, we can say that $\theta(r, \pi)<\Theta$, and (ii) for a feasible contract $d=r \cdot c-\pi \cdot c(\pi) \geq 0$ which implies that $\theta(r, \pi)>0$. The fact that $\theta \in[0, \Theta]$ induces therefore no additional constraints.

${ }^{3}$ Since we have assumed that our measure is absolutely continuous, we decide by convention to only use weak inequalities.
} 
Remark 1 Since the total surplus under an $A D F$ system is given $\int_{c}^{A} \int_{0}^{\Theta} u_{\bar{w}}^{(\alpha, \theta)} d F$, we can say that these households do not get worse after the introduction of a $w A D F$. Moreover if $\theta(r, \pi)>0$, we can even say that this average surplus increases when we move from an ADF to a wADF system. This last case occurs when the budget balancing price cut $d=c \cdot r-\pi \cdot c(\pi)$ is strictly positive.

Let us now move to the agents who really gain from the introduction of a waste management contract. These households were initially, say, too "poor" to buy the good (i.e. $\alpha<c$ ) but are nevertheless "environment friendly" (i.e. $\theta$ small). These consumers are ready to spend some time to reduce her waste contain and, by doing so, reduce her cost of acquisition of the good. This set of $N$ ew buyers is therefore given by :

$$
N(r, \pi)=\left\{(\alpha, \theta) \in[0, A] \times[0, \Theta]: 0 \leq \alpha \leq c \text { and } u_{w e}^{(\alpha, \theta)}(r, \pi) \geq 0\right\}
$$

Now let us observe that the second condition is equivalent to $\alpha \geq \theta \cdot e(r)+\pi \cdot c(\pi)+(1-r) \cdot c \geq 0$. But for these agents, $\alpha$ is bounded from above by $c$, we can therefore introduce the function $\alpha(r, \pi, \theta):=\frac{\theta r}{\bar{r}}+c-(r c-\pi c(\pi))$ with $\theta \in[0, \theta(r, \pi)]$ and say that :

$$
N(r, \pi)=\{(\alpha, \theta) \in[0, A] \times[0, \Theta]: \alpha(r, \pi, \theta) \leq \alpha \leq c \text { and } 0 \leq \theta \leq \theta(r, \pi)\}
$$

The average surplus of these new consumers is therefore given by :

$$
\begin{aligned}
S_{N}(r, \pi) & =\int_{C(r, \pi)} u_{w e}^{(\alpha, \theta)}(r, \pi) d F \\
& =\int_{0}^{\theta(r, \pi)}\left(\int_{\alpha(r, \pi, \theta)}^{c} u_{w e}^{(\alpha, \theta)}(r, \pi) f(\alpha / \theta) d \alpha\right) f(\cdot, \theta) d \theta
\end{aligned}
$$

and we again observe that :

Remark 2 From the previous remark, we know that for a strictly positive budget balancing price cut we have $\theta(r, \pi)>0$ and under the same condition, we also observe that $\forall \theta<\theta(r, \pi)$, $\alpha(r, \pi, \theta)<0$. We can therefore assert that if the price cut $c \cdot r-\pi \cdot c(\pi)>0$ then $S_{N}(r, \pi)>0$, in other words there is a second gain induced by the introduction of an wADF system.

Let us now consider the study of the properties of the total surplus $S(r, \pi):=S_{I}(r, \pi)+$ $S_{N}(r, \pi)$ on the set $\mathcal{F}$ of feasible contracts. If the monitoring probability $\pi$ increases, the budget balancing price cut $d=c \cdot r-\pi \cdot c(\pi)$ automatically decreases. We can therefore say, at least 
from an intuitive point of view, that the welfare of the consumers who have adopted the waste management contract decreases. The effect of a change of the required waste treatment rate $r$ is however less obvious. On the one hand, an increase in $r$ contributes to a higher price cut $d$. This provides, for the households who buy the good, more incentives to accept the contract and gives the opportunity to new consumers to enter the market. Yet, on the other hand, this also implies that the consumers who accept the contract provide a higher level of effort. We nevertheless show that this increase of the effort is compensated by the increase of the price cut. More formally, we say that :

Proposition 2 Let us denote by $P(r, \pi):=\int_{0}^{\theta(r, \pi)} \int_{\alpha(r, \pi, \theta)}^{A} d F$ the proportion of households who accept the waste management contract and by $\bar{\Theta}(r, \pi):=\int_{0}^{\theta(r, \pi)} \int_{\alpha(r, \pi, \theta)}^{A} \theta \frac{d F}{P(r, \pi)}$ the average desutility of the effort for the households who accept the waste management contract. We observe that :

(i) $\forall(r, \pi) \in \mathcal{F}$ and $(r, \pi) \neq(0,0), \partial_{\pi} S(r, \pi)=-\left(\pi c^{\prime}(\pi)+c(\pi)\right) P(r, \pi)<0$, i.e. when the probability of control increases, the consumers surplus decreases.

(ii) $\forall(r, \pi) \in \mathcal{F}$ and $(r, \pi) \neq(0,0), \partial_{r} S(r, \pi)=\left(c-\frac{\bar{\Theta}(r, \pi)}{\bar{r}}\right) P(r, \pi)>0$ i.e. the surplus increases with the required waste treatment rate $r$ since for all consumers who accept the contract the price cut, $c \cdot r-\pi \cdot c(\pi)$ is always greater than the monetary evaluation of the effort $\theta \frac{r}{\bar{r}}$, so that $c>\frac{\theta}{\bar{r}}$ and also on average.

\section{The optimal wADF strategy}

We can finally have a look at the description of the optimal wADF policy. As usual in a second best situation induced by the existence of illegal dumping, the social planner tries to implement the wADF feasible contract which maximizes the total surplus, i.e. she chooses :

$$
\left(r^{*}, \pi^{*}\right) \in \underset{(r, \pi) \in \mathcal{F}}{\arg \max } \underbrace{\int_{C(r, \pi) \cup N(r, \pi)} u_{w e}^{(\alpha, \theta)}(r, \pi) d F+\int_{\bar{C}(r, \pi)} u_{\bar{w}}^{(\alpha, \theta)} d F}_{S(r, \pi)}
$$

The reader immediately observes that this program admits a solution. In fact, $\mathcal{F}$ is a closed subset of the compact set $[0, \bar{r}] \times[0,1]$, hence is compact and $S(r, \pi)$ is a continuous function. So let us now characterize this optimum.

First, let us remember, from proposition 1, that the wADF contracts which are budget balancing, incentive compatible, and satisfies the participation contraint are such that :

$$
\frac{\pi \cdot c(\pi)}{c} \leq r \leq \min \left\{\frac{c_{d}}{c}+\frac{\pi \cdot\left(c(\pi)+c_{S}\right)}{c}, \bar{r}\right\}
$$


But from proposition 2, we also know that the average surplus is (i) increasing with $r$ and (ii) decreasing with $\pi$. This immediately implies that (i) an optimal wADF policy has the property that $r^{*}=\min \left\{\frac{c_{d}}{c}+\frac{\pi^{*} \cdot\left(c\left(\pi^{*}\right)+c_{S}\right)}{c}, \bar{r}\right\}$ and (ii) this policy cannot be such that $\frac{c_{d}}{c}+\frac{\pi^{*} \cdot\left(c\left(\pi^{*}\right)+c_{S}\right)}{c}>\bar{r}$ otherwise the policy maker has the ability to reduce $\pi$ without changing $r$. It follows that :

$$
r^{*}=\frac{c_{d}}{c}+\frac{\pi^{*} \cdot\left(c\left(\pi^{*}\right)+c_{S}\right)}{c}
$$

If we now set $r_{\text {inf }}:=\frac{c_{d}}{c}$ and construct ${ }^{4} \pi_{\text {sup }}$ the probabilty of control which satisfies $\frac{c_{d}}{c}+$ $\frac{\pi_{\text {sup }} \cdot\left(c\left(\pi_{\text {sup }}\right)+c_{S}\right)}{c}=\bar{r}$, we can say that :

Proposition 3 Any optimal wADF policy $\left(r^{*}, \pi^{*}\right)$ has the properties that :

(i) $r^{*} \geq r_{\mathrm{inf}}>0$, i.e. this system always induces a waste reduction effort on the consumers side contrary to an ADF system. This property is even independent from the monitoring costs.

(ii) $\pi^{*} \leq \pi_{\text {sup }}$, i.e. the probability of control and therefore the monitoring costs are bounded from above. Moreover this upper bound decreases with both the illegal disposal cost $c_{d}$ and the social cost $c_{s}$ of illegal dumping.

(iii) $c \cdot r^{*}-\pi^{*} \cdot c\left(\pi^{*}\right)=c_{d}+\pi^{*} \cdot c_{S}$, i.e the price cut obtained by the consumers is equal to the total cost of illegal dumping (including the fine) so that the agents are indifferent between an illegal dumping strategy and a legal disposal one without any effort.

(iv) $d^{*}=c \cdot r^{*}-\pi^{*} \cdot c\left(\pi^{*}\right) \geq c_{d}>0$, i.e. the price cut is strictly positive, hence by remarks 1 and 2 the wADF policy striclty dominated the ADF system and "poorer environment friendly" consumers always enter the market.

If we want to go a step further in the characterization of an optimal wADF policy, let us take advantage of point (iii) of the preceding proposition. In fact, since the price cut must be equal to the global cost of illegal dumping, i.e.

$$
f(r, \pi)=c \cdot r-\pi \cdot c(\pi)-\left(c_{d}+\pi \cdot c_{S}\right)=0
$$

we can, by the implicite function theorem construct a relation between the probality of control and the required waste management rate. More precisely we can say :

Remark 3 There exists a function ${ }^{5} \pi:\left[r_{\text {inf }}, \bar{r}\right] \rightarrow\left[0, \pi_{\text {sup }}\right]$ with the properties that (i) $f(r, \pi(r))=$ 0 , (ii) $\frac{d \pi}{d r}=\frac{c}{\pi \cdot c^{\prime}(\pi)+\left(c(\pi)+c_{S}\right)}$ and (iii) the graph of $\pi$ belongs to $\mathcal{F}$. It follows that the program

\footnotetext{
${ }^{4}$ Since $\frac{\pi \cdot\left(c(\pi)+c_{S}\right)}{c}$ is increasing and $c(1)>c$, the reader observes that the probability $\pi_{\text {sup }}$ is defined.

${ }^{5}$ The reader surrely remarks that our technical assumption which says that $\frac{c_{d}}{c}<\bar{r}$ becomes crucial now. If this assumption is not satisfied, the only optimal policy would be to set $r^{*}=\bar{r}$ and $\pi^{*}=\pi_{\text {sup }}=0$.
} 
given by equation (1) can be reduced to $r^{*} \in \underset{r_{\text {inf }} \leq r \leq \bar{r}}{\arg \max } S(r, \pi(r))$.

Since we know from proposition 2 the derivates of $S(r, \pi)$, we can even say that :

Proposition 4 An optimal wADF policy $\left(r^{*}, \pi^{*}\right)$ satisfies :

$$
\left(c-\frac{\bar{\Theta}\left(r^{*}, \pi^{*}\right)}{\bar{r}}\right)-\left.\left(\pi^{*} \cdot c^{\prime}\left(\pi^{*}\right)+c\left(\pi^{*}\right)\right) \cdot \frac{d \pi}{d r}\right|_{r^{*}} \geq 0 \text { with equality when } r^{*}<\bar{r}
$$

This result tells us first that the optimal waste treatment rate $r^{*}$ is strictly greater than $r_{\text {inf }}$. In fact, from remark 3 , we know that the probability of control $\pi\left(r_{\text {inf }}\right)$ associated to $r_{\text {inf }}$ is nought so that the marginal monitoring cost $\pi^{*} \cdot c^{\prime}\left(\pi^{*}\right)+c\left(\pi^{*}\right)=0$. But the average net marginal gain of the consumers who accept the contract, ie. $\left(c-\frac{\bar{\Theta}\left(r_{\text {inf }}, 0\right)}{\bar{r}}\right)$ is strictly positive since the upper bound of $\theta$ on which this average value is computed is $\bar{r} \cdot c$.

From that point of view, the social planner has always an incentive to increase the optimal waste treatment rate above $r_{\text {inf }}$, but each rate $r>r_{\text {inf }}$ requires an active monitoring policy that prevents illegal dumping. The control costs then reduces, by the budget balancing constraint, the price cut obtained by the consumers who are willing to participate in the waste management program. It follows that the social planner will try to set the optimal waste treatment rate $r^{*}$ correspond to the average marginal gain minus the marginal cost induced by an increase of the required effort, i.e the quantity $\left(c-\frac{\bar{\Theta}\left(r^{*}, \pi^{*}\right)}{\bar{r}}\right)$ is equal (or at least greater then ) to the marginal cost of monitoring induced by a higher waste treatment rate., i.e. the quantity $\left.\left(\pi^{*} \cdot c^{\prime}\left(\pi^{*}\right)+c\left(\pi^{*}\right)\right) \cdot \frac{d \pi}{d r}\right|_{r^{*}}$.

\section{Concluding remarks}

In any case, we have shown in this paper that a policy maker never has an interest in implement a crude ADF system on a given market . If she does it, she definitely annihilates any incentives, at a household level, to participate in a waste management program. What's more, she excludes from this market some poorer consumers who are ready to participate in this program in order to reduce the total cost of their consumption i.e. including the waste management cost. That is why we proposed to wave this ADF system by introducing a contract which specify a waste treatment target in counterpart to a lower disposal fee. We even couple this contract with a monitoring technology in order to prevent illegal dumping. In this context, we have checked that feasable contracts, i.e. satisfying a Budget Balancing, an Incentive and a Participation constraints, exist and that an optimal contract in this set always increases the welfare of the consumers in respect to a simple ADF system. 
This paper however remains particular in several respects. First of all, we have mainly focused our attention on the households and looked at the waste management policy as a treatment of "end-of-pipe" pollution. So by highlighting our main argument, we neglect not only the question of the incentives which could be given to the firms in order to reduce the waste contain of their products but also the existence of potential recycling methods which lead to a partial reintroduction of the waste in the production process. We leave these points to future works.

As usual in this litterature, we have also assumed that the market of the good under consideration works competitively. The results surely change under imperfect competition. In this case, the optimal design of the contract should not only take into account the waste management issue but also the effects of this contract on the market power. More preciselly we have shown that a wADF system dominates a ADF policy since new consumers have the opportunity to enter the market. Yet, in a context of imperfect competition this also modifies the elasticity of the demand and has therefore an impact on the market power.

Finally, even if our argument requires no specific assumption on the distribution of the characteristics though agents and remains quite general from that point of view, we have assumed that the relation between the effort and the cost reduction rate is linear. It could perhaps be interesting to consider more general functions which characterize this relation.

\section{References}

[1] Calcott, P. and M. Walls, 2000, 'Can downstream Waste Disposal Policies encourage upstream "Design for Environement" ?', American Economic Review : 90, 233-237.

[2] Calcott, P. and M. Walls, 2005, 'Waste Recycling and "Design for Environement" : Role for Market and Policy Instruments', Ressource and Energy Economics : 27, 287-305.

[3] Choe, C. and I. Fraser, 1999, 'An Economic Analysis of Household Waste Management', Journal of Environmental Economics and Management, 38, 234-246.

[4] Choe, C. and I. Fraser, 2001, 'On the Flexibility of Optimal Policies for Green Design', Environmental and Ressource Economics, 18, 367-371.

[5] Dinan, T.M., 1993, 'Economic Efficiency Effects of Alternative Policies for Reducing Waste Disposal', Journal of Environmental Economics and Management, 25, 242-256. 
[6] Fullerton, D. and T. C. Kinnaman, 1995, 'Garbadge, Recycling and Illicit Burning or Dumping', Journal of Environmental Economics and Management, 29, 78-91.

[7] Fullerton, D. and T. C. Kinnaman, 1996, 'Household Responses to Pricing Garbage by the Bag', American Economic Review, 86, 971-984.

[8] Fullerton, D. and W. Wu, 1998, 'Policies for Green Design', Journal of Environmental Economics and Management, 36, 131-148.

[9] Jenkins, R.R., Robin R., 1993, "The Economics of Solid Waste Reduction", Hants, Edward Elgar Publishing Limited.

[10] Jenkins, R.R., S.A. Martinez, K. Palmer and M.J. Podolsky, 2003, 'The Determinant of Household Recycling : a Material-Specific Analysis of Recycling Program Features and Unit Princing', Journal of Environmental Economics and Management, 45, 294-318.

[11] Palmer, K. and M. Walls, 1997, 'Optimal policies for Solid Waste Disposal Taxes, Subsidies and Standards', Journal of Public Economics 65 : 193-205.

[12] Palmer, K., H. Sigman and M. Walls, 1997, 'The Cost of Reducing Municipal Solid Waste', Journal of Environmental Economics and Management, 33, 128-150.

[13] Shinkuma, T., 2003, 'On the Second-Best Policy of Household's Waste Recycling', Environmental and Ressource Economics, 24, 77-95.

[14] Shinkuma, T., 2007, 'Reconsideration of an Advance Disposal Fee Policy for End-of-Pipe Durable Goods', Journal of Environmental Economics and Management 53 : 110-121.

[15] Walls, M. and K. Palmer, 2001, 'Upstream Pollution, Dowstream Waste Disposal and the Design of Compréhensive Environmental Policies', Journal of Environmental Economics and Management, 41, 94-108.

\section{APPENDIX}

\section{A Proof of lemma 1}

Obvious 


\section{B Proof of lemma 2}

Let us first observe that if $r \cdot c-\pi \cdot c(\pi) \geq 0$ then for $\theta=0$, we have :

$$
u_{w e}^{(\alpha, 0)}(r, \pi)=\alpha-c+r \cdot c-\pi \cdot c(\pi) \geq \alpha-c=u_{\bar{w}}^{(\alpha, 0)}
$$

Since $A>c$, it remains to choose $\alpha=A$ in order to observe that $u_{w e}^{(A, 0)}(r, \pi) \geq u_{\bar{w}}^{(A, 0)} \geq 0$. Without illegal dumping there exists therefore $(\alpha, \theta)=(A, 0)$ such that $\mathrm{PC}$ is true.

Now let us suppose that $r \cdot c-\pi \cdot c(\pi)<0$, then $\forall \theta \in[0, \Theta], r \cdot c-\pi \cdot c(\pi)-\theta \frac{r}{\bar{r}}<0$. Since $e(r)=\frac{r}{\bar{r}}$, this implies $\forall(\alpha, \theta) \in[0, A] \times[0, \Theta]$,

$$
u_{w e}^{(\alpha, \theta)}(r, \pi)=\alpha-c+r \cdot c-\pi \cdot c(\pi)-\theta e(r)<\alpha-c=u_{\bar{w}}^{(\alpha, \theta)}
$$

By the definition of a maximum, this also means that :

$$
\forall(\alpha, \theta) \in[0, A] \times[0, \Theta], \max \left\{0, u_{\bar{w}}^{(\alpha, \theta)}, u_{w e}^{(\alpha, \theta)}(r, \pi), u_{w \bar{e}}^{(\alpha, \theta)}(r, \pi)\right\}>u_{w e}^{(\alpha, \theta)}(r, \pi)
$$

But this contradicts $(\mathrm{PC})$

\section{Proof of lemma 3}

If $r \cdot c \leq c_{d}+\pi \cdot c_{s}+\pi \cdot c(\pi)$ holds then

$$
\forall \alpha \in[0, A] \quad u_{\bar{w}}^{(\alpha, \theta)}=\alpha-c \geq \alpha-(1-r) \cdot c-\pi \cdot c(\pi)-c_{d}-\pi c_{s}=u_{w \bar{e}}^{(\alpha, \theta)}(r, \pi)
$$

and, by the definition of a maximum, we can conclude that :

$$
\forall(\alpha, \theta) \in[0, A] \times[0, \Theta], \quad \max \left\{u_{w e}^{(\alpha, \theta)}(r, \pi), u_{\bar{w}}^{(\alpha, \theta)}, 0\right\} \geq u_{w \bar{e}}^{(\alpha, \theta)}(r, \pi)
$$

Now assume that $r \cdot c>c_{d}+\pi \cdot c_{s}+\pi \cdot c(\pi)$. By a similar argument as before, we can now say that $\forall \alpha \in[0, A]$, $u_{\bar{w}}^{(\alpha, \theta)}<u_{w \bar{e}}^{(\alpha, \theta)}(r, \pi)$. Since $c<A$, if we set $\alpha=A$, we can say that :

$$
\forall \theta \in[0, \Theta], \quad u_{w \bar{e}}^{(A, \theta)}(r, \pi)>\max \left\{u_{\bar{w}}^{(A, \theta)}, 0\right\}
$$

Moreover under the same assumption we have that $r \cdot c>c_{d}+\pi \cdot c_{s}$ since $\pi \cdot c(\pi) \geq 0$. It follows that $\forall \theta \in[0, \Theta]$,

$$
A-(1-r) \cdot c-\pi \cdot c(\pi)-\left(c_{d}+\pi \cdot c_{s}\right)>A-(1-r) \cdot c-\pi \cdot c(\pi)-\frac{r}{\bar{r}} \cdot(\bar{r} \cdot c)
$$

Since $\Theta>c, e(r)=\frac{r}{\bar{r}}$ and $\bar{r} \leq 1$, we deduce that

$$
u_{w \bar{e}}^{(A, \Theta)}(r, \pi)=A-(1-r) \cdot c-\pi \cdot c(\pi)-\left(c_{d}+\pi \cdot c_{s}\right)>A-(1-r) \cdot c-\pi \cdot c(\pi)-\Theta \cdot e(r)=u_{w e}^{(A, \Theta)}(r, \pi)
$$

By putting all the previous results together, we conclude that :

$$
\exists(\alpha, \theta)=(A, \Theta), \quad u_{w \bar{e}}^{(A, \Theta)}(r, \pi)>\max \left\{u_{w e}^{(A, \Theta)}(r, \pi), u_{\bar{w}}^{(A, \Theta)}, 0\right\}
$$

But this contradicts (IC) 


\section{Proof of proposition 1}

This is a direct consequence of lemmata 1,2 and 3

\section{E Proof of proposition 2}

This computation is a tedious exercise since $S(r, \pi)=S_{I}(r, \pi)+S_{N}(r, \pi)$ with

$$
\left\{\begin{array}{l}
S_{I}(r, \pi)=\int_{0}^{\theta(r, \pi)}\left(\int_{c}^{A} u_{w e}^{(\alpha, \theta)}(r, \pi) \cdot f(\alpha / \theta) d \alpha\right) f(\cdot, \theta) d \theta+\int_{\theta(r, \pi)}^{\Theta}\left(\int_{c}^{A} u_{\bar{w}}^{(\alpha, \theta)} \cdot f(\alpha / \theta) d \alpha\right) f(\cdot, \theta) d \theta \\
S_{N}(r, \pi)=\int_{0}^{\theta(r, \pi)}\left(\int_{\alpha(r, \pi, \theta)}^{c} u_{w e}^{(\alpha, \theta)}(r, \pi) f(\alpha / \theta) d \alpha\right) f(\cdot, \theta) d \theta
\end{array}\right.
$$

But the reader observes that $r$ and $\pi$ work in a rather similar way. So if $x$ stand either for $r$ and $\pi$, we obtain that:

$$
\begin{aligned}
\partial_{x} S(r, \pi) & =\partial_{x} S_{I}(r, \pi)+\partial_{x} S_{N}(r, \pi)= \\
& \left.\left(\left(\int_{c}^{A} u_{w e}^{(\alpha, \theta)}(r, \pi) \cdot f(\alpha / \theta) d \alpha\right) f(\cdot, \theta)\right)\right|_{\theta=\theta(r, \pi)} \times \partial_{x} \theta(r, \pi) \\
& +\int_{0}^{\theta(r, \pi)}\left(\int_{c}^{A} \partial_{x} u_{w e}^{(\alpha, \theta)}(r, \pi) \cdot f(\alpha / \theta) d \alpha\right) f(\cdot, \theta) d \theta \\
& -\left.\left(\left(\int_{c}^{A} u_{\bar{w}}^{(\alpha, \theta)} \cdot f(\alpha / \theta) d \alpha\right) f(\cdot, \theta)\right)\right|_{\theta=\theta(r, \pi)} \times \partial_{x} \theta(r, \pi) \\
& \left.+\left(\int_{\alpha(r, \pi, \theta)}^{c} u_{w e}^{(\alpha, \theta)}(r, \pi) f(\alpha / \theta) d \alpha\right) f(\cdot, \theta)\right)\left.\right|_{\theta=\theta(r, \pi)} \times \partial_{x} \theta(r, \pi) \\
& +\int_{0}^{\theta(r, \pi)}\left(\int_{\alpha(r, \pi, \theta)}^{c} \partial_{x} u_{w e}^{(\alpha, \theta)}(r, \pi) f(\alpha / \theta) d \alpha-\left.\left(u_{w e}^{(\alpha, \theta)}(r, \pi) f(\alpha / \theta)\right)\right|_{\alpha=\alpha(r, \pi, \theta)} \partial_{x} \alpha(r, \pi, \theta)\right) f(\cdot, \theta) d \theta
\end{aligned}
$$

Since $\theta(r, \pi)=\bar{r}\left(c-\frac{\pi \cdot c(\pi)}{r}\right)$ and $\alpha(r, \pi, \theta):=\frac{\theta r}{\bar{r}}+c-(r c-\pi c(\pi))$, we also remark that :

$$
\left\{\begin{array}{l}
\text { (i) }\left.u_{w e}^{(\alpha, \theta)}(r, \pi)\right|_{\theta=\theta(r, \pi)}=\alpha-(1-r) \cdot c-\pi \cdot c(\pi)-\bar{r}\left(c-\frac{\pi \cdot c(\pi)}{r}\right) \frac{r}{\bar{r}}=\alpha-c=\left.u_{\bar{w}}^{(\alpha, \theta)}\right|_{\theta=\theta(r, \pi)} \\
\text { (ii) }\left.\alpha(r, \pi, \theta)\right|_{\theta=\theta(r, \pi)}=\bar{r}\left(c-\frac{\pi \cdot c(\pi)}{r}\right) \frac{r}{\bar{r}}+c-(r c-\pi c(\pi))=c \\
\text { (iii) }\left.u_{w e}^{(\alpha, \theta)}(r, \pi)\right|_{\alpha=\alpha(r, \pi, \theta)}=\theta \frac{r}{\bar{r}}+c-(r c-\pi c(\pi))-(1-r) \cdot c-\pi \cdot c(\pi)-\theta \frac{r}{\bar{r}}=0
\end{array}\right.
$$

By (i) the first (2a) and the third (2c) term in the preceding sum simplify, by (ii) the fourth term (2d) is zero, and (iii) simplifies the last term (2e). We can therefore say that:

$$
\partial_{x} S(r, \pi)=\int_{0}^{\theta(r, \pi)}\left(\int_{\alpha(r, \pi, \theta)}^{A} \partial_{x} u_{w e}^{(\alpha, \theta)}(r, \pi) \cdot f(\alpha / \theta) d \alpha\right) f(\cdot, \theta) d \theta
$$

Now remember that $\partial_{\pi} u_{w e}^{(\alpha, \theta)}(r, \pi)=-\left(\pi c^{\prime}(\pi)+c(\pi)\right)<0$ for all $\pi>0$ and that the only feasible contract for which $\pi=0$ is $(r, \pi)=(0,0)$, we can therefore say that :

$$
\forall(r, \pi) \in \mathcal{F} \text { and }(r, \pi) \neq(0,0), \quad \partial_{\pi} S(r, \pi)=-\left(\pi c^{\prime}(\pi)+c(\pi)\right) \int_{0}^{\theta(r, \pi)} \int_{\alpha(r, \pi, \theta)}^{A} d F<0
$$


where $\int_{0}^{\theta(r, \pi)} \int_{\alpha(r, \pi, \theta)}^{A} d F=P(r, \pi)$ the proportion of households who accept the waste management contract. This proves (i) of proposition 2.

Let us move to (ii) of proposition 2. Since $e(r)=\frac{r}{\bar{r}}$, we observe that $\partial_{r} u_{w e}^{(\alpha, \theta)}(r, \pi)=c-\frac{\theta}{\bar{r}}$. Moreover $\forall \theta<\theta(r, \pi)=: \bar{r}\left(c-\frac{\pi \cdot c(\pi)}{r}\right)$, we can say that :

$$
c-\frac{\theta}{\bar{r}}>c-\bar{r}\left(c-\frac{\pi \cdot c(\pi)}{r}\right) \frac{1}{\bar{r}}=\frac{\pi \cdot c(\pi)}{r} \geq 0
$$

This implies that :

$$
\forall(r, \pi) \in \mathcal{F} \text { and }(r, \pi) \neq(0,0), \quad \partial_{r} S(r, \pi)=\int_{0}^{\theta(r, \pi)}\left(c-\frac{\theta}{\bar{r}}\right)\left(\int_{\alpha(r, \pi, \theta)}^{A} f(\alpha / \theta) d \alpha\right) f(\cdot, \theta) d \theta>0
$$

Now let us denote by

$$
\bar{\Theta}(r, \pi):=\int_{0}^{\theta(r, \pi)} \theta \frac{\left(\int_{\alpha(r, \pi, \theta)}^{A} f(\alpha / \theta) d \alpha\right)}{\int_{0}^{\theta(r, \pi)} \int_{\alpha(r, \pi, \theta)}^{A} d F} f(\cdot, \theta) d \theta
$$

the average desutility of the effort for the households who accept the waste management contract. This yields :

$$
\forall(r, \pi) \in \mathcal{F} \text { and }(r, \pi) \neq(0,0), \quad \partial_{r} S(r, \pi)=\left(c-\frac{\bar{\Theta}(r, \pi)}{\bar{r}}\right) P(r, \pi)
$$

\section{F $\quad$ Proof of proposition 3}

Obvious

\section{G Proof of proposition 4}

By remark 3, we know that the search of an optimal contract reduces to the search of a waste reduction rate which satisfies

$$
r^{*} \in \underset{r_{\text {inf }} \leq r \leq \bar{r}}{\arg \max } S(r, \pi(r)) .
$$

From that point of view any optimal solution satisfies the following Kuhn-Tucker first order conditions :

$$
\left\{\begin{array}{l}
\partial_{r} S(r, \pi(r))+\partial_{\pi} S(r, \pi(r)) \frac{d \pi}{d r}+\lambda_{1}-\lambda_{2}=0 \\
\lambda_{1}\left(r_{\min }-r\right)=0 \quad r_{\min }-r \leq 0 \quad \lambda_{1} \geq 0 \\
\lambda_{2}(r-\bar{r})=0 \quad r-\bar{r} \leq 0 \quad \lambda_{2} \geq 0
\end{array}\right.
$$

where $\lambda_{1}$ and $\lambda_{2}$ are the Lagrangian multiplicators associated to the constraints $r_{\min }-r \leq 0$ and $r-\bar{r} \leq 0$ and

$$
\begin{aligned}
\partial_{r} S(r, \pi) & =\left(c-\frac{\bar{\Theta}(r, \pi)}{\bar{r}}\right) P(r, \pi) \\
\partial_{\pi} S(r, \pi) & =-\left(\pi c^{\prime}(\pi)+c(\pi)\right) P(r, \pi) \\
\frac{d \pi}{d r} & =\frac{c}{\pi \cdot c^{\prime}(\pi)+\left(c(\pi)+c_{S}\right)}
\end{aligned}
$$


Since we have assumed that $\frac{c_{d}}{c}<\bar{r}$ both constraints cannot be binding. So if the first constraint is binding, i.e. $r^{*}=r_{\min }$ then $\lambda_{2}=0$. But in this case $\pi^{*}=\pi\left(r_{\min }\right)=0$ and the first order condition becomes :

$$
\left(c-\frac{\bar{\Theta}\left(r_{\min }, 0\right)}{\bar{r}}\right) P\left(r_{\min }, 0\right)+\lambda_{1}=0
$$

Let us now observe that $\theta\left(r_{\min }, 0\right):=\bar{r} c>0$. This implies that $P\left(r_{\min }, 0\right)=\int_{0}^{\bar{r} c} \int_{\frac{\theta r_{\min }}{\bar{r}}+c\left(1-r_{\min }\right)}^{A} d F \geq$ $\int_{0}^{\bar{r} c} \int_{c}^{A} d F>0$ and that $\bar{\Theta}\left(r_{\min }, 0\right):=\int_{0}^{\bar{r} c} \theta d G<\bar{r} c$ where $G(\theta)=$ probability $[t \leq \theta /$ contract is accepted $]$. It follows that $\lambda_{1}<0$ which is a contradiction.

Let us now move to a pure interior solution. In this case $\left.r^{*} \in\right] r_{\min }, \bar{r}\left[\right.$ and $\lambda_{1}=\lambda_{2}=0$. it follows that :

$$
\left(c-\frac{\bar{\Theta}\left(r^{*}, \pi^{*}\right)}{\bar{r}}-\left(\pi^{*} c^{\prime}\left(\pi^{*}\right)+c\left(\pi^{*}\right)\right) \frac{d \pi}{d r}\right) P\left(r^{*}, \pi^{*}\right)=0
$$

But by proposition 3, we know that the optimal price cut $d^{*}=r^{*} c-\pi^{*} c\left(\pi^{*}\right)$ is strictly positive. As a consequence we can say that at the optimum $\theta\left(r^{*}, \pi^{*}\right):=\bar{r}\left(c-\frac{\pi^{*} c\left(\pi^{*}\right)}{r^{*}}\right)>0$ and $\forall \theta \leq \theta\left(r^{*}, \pi^{*}\right), \alpha\left(r^{*}, \pi^{*}, \theta\right):=\frac{\theta r^{*}}{\bar{r}}+c-$ $\left(r^{*} c-\pi^{*} c\left(\pi^{*}\right)\right) \leq c$. This implies that $P\left(r^{*}, \pi^{*}\right):=\int_{0}^{\theta\left(r^{*}, \pi^{*}\right)} \int_{\alpha\left(r^{*}, \pi^{*}, \theta\right)}^{A} d F \geq \int_{0}^{\theta\left(r^{*}, \pi^{*}\right)} \int_{c}^{A} d F>0$. So if the optimal strategy $\left.r^{*} \in\right] r_{\min }, \bar{r}[$ we have :

$$
c-\frac{\bar{\Theta}\left(r^{*}, \pi^{*}\right)}{\bar{r}}-\left(\pi^{*} c^{\prime}\left(\pi^{*}\right)+c\left(\pi^{*}\right)\right) \frac{d \pi}{d r}=0
$$

It remains to consider the last case in which the second constraint is binding (i.e. $r^{*}=\bar{r}$ ). This implies that (i) $\pi^{*}=\pi(\bar{r})=\pi_{\text {sup }}$ and (ii) $\lambda_{1}=0$ since both constraints cannot be binding. Moreover with a same argument as before we can say that $P\left(r^{*}, \pi^{*}\right)>0$. Since $\lambda_{2} \geq 0$, we can conclude that if $\left(r^{*}, \pi^{*}\right)=\left(\bar{r}, \pi_{\sup }\right)$ is an optimum then

$$
c-\frac{\bar{\Theta}\left(\bar{r}, \pi_{\text {sup }}\right)}{\bar{r}}-\left(\pi_{\text {sup }} c^{\prime}\left(\pi_{\text {sup }}\right)+c\left(\pi_{\text {sup }}\right)\right) \frac{d \pi}{d r}=\lambda_{2} \geq 0
$$

By putting together all these results, we can conclude that an optimal wADF policy $\left(r^{*}, \pi^{*}\right)$ satisfies :

$$
\left(c-\frac{\bar{\Theta}\left(r^{*}, \pi^{*}\right)}{\bar{r}}\right)-\left.\left(\pi^{*} \cdot c^{\prime}\left(\pi^{*}\right)+c\left(\pi^{*}\right)\right) \cdot \frac{d \pi}{d r}\right|_{r^{*}} \geq 0 \text { with equality when } r^{*}<\bar{r}
$$

